\title{
Das koptische Christentum
}

(Auswärtige Sitzung der Akademie der Wissenschaften am 8. Juli 2011 im Koptischen Kloster Brenkhausen)

\author{
MARTIN TAMCKE
}

\section{Einleitung}

Kopte - das heißt soviel wie Ägypter. ${ }^{1}$ Wer sich dazu bekennt, Kopte zu sein, der hebt innerhalb Ägyptens auf Unterscheidung ab. Die koptische Sprache entstand im 3. Jahrhundert; sie ist nur für die Spätantike und das Mittelalter eines der Kennzeichen der koptisch-christlichen Kultur. Nach den Aufständen der Kopten gegen die arabisch-muslimische Herrschaft wurde sie vom Arabischen verdrängt, auch im täglichen Gebrauch der Kopten. ${ }^{2}$

Man kann den Kopten bis heute einen Vorwurf daraus machen, dass sie sich damals nicht als Araber verstanden und sich vielfach auch heute nicht als Araber verstehen. Prinz Hassan von Jordanien etwa findet kritische Worte dazu in seinem Buch über die orientalische Christenheit. ${ }^{3}$ Solches Befremden denen gegenüber, die sich nicht assimilieren wollen, ist heute ganz üblich in weiten Kreisen der einfachen arabisch-muslimischen Bevölkerung Ägyptens. Kopten, das sind für nicht wenige die „Anderen“ zum „Eigenen“. Eine kleine Episode aus der Binnenrezeption der Kopten mag das illustrieren:

Da sitzt einer mit fünf Kopten zusammen bei einem Essen. Beim Zwischengang wird einer übergangen. Der Übergangene hat Humor und fragt

$1 \quad$ Allgemeine Literatur: A. Gerhards/H. Brakmann, Die koptische Kirche, Stuttgart 1994; W. Reiss, Erneuerung in der Koptisch-Orthodoxen Kirche, Hamburg 1998; W. Boochs, Geschichte und Geist der Koptischen Kirche, Langwarden 2004; O. F. A. Meinardus, Christians in Egypt, Kairo/New York 2006; W. Hage, Das orientalische Christentum, Stuttgart 2007; C. Lange/K. Pinggéra, Die altorientalischen Kirchen, Darmstadt 2010. Als klassische Selbstdarstellung aus orientalisch-orthodoxer Feder, aber heute vielfach ungenügend: P. Verghese, Koptisches Christentum, Die orthodoxen Kirchen Ägyptens und Äthiopiens, Stuttgart 1973.

2 Vgl. M. Tamcke, Christen in der islamischen Welt. Von Mohammed bis zur Gegenwart, München 2008, 71-75.

3 El Hassan bin Talal, Das Christentum in der arabischen Welt, Wien 2003. 
ironisch: „Why me? (Warum ich?)“. Ein anderer Kopte antwortet: „Weil Du ein Kopte bist!“ Lautes Gelächter. ${ }^{4}$ Ein Witz, der unter Insidern funktioniert. Ein Witz, der es auf den Punkt bringt, meint der deutsche Journalist, der die Episode erzählt. Kopten werden benachteiligt. Das hat weit in die Geschichte zurückreichende Gründe.

Es gibt aber auch immer noch den anderen Aspekt der Existenz von christlichen Kopten in Ägypten: sie verhindern allein schon durch ihre Präsenz, dass Ägypten zu einem ausschließlich muslimischen Land wird, das dann endgültig seine große multireligiöse und multikulturelle Vergangenheit für die Gegenwart vernichtet hätte. Noch vor etwas über hundert Jahren fuhr ein Philosoph wie Solowjew nur deshalb nach Ägypten, weil dieses Land ihm und vielen Zeitgenossen als Hort des Multireligiösen und Synkretistischen galt. ${ }^{5}$ Doch die Anwesenheit der Kopten schuf in Ägypten nicht nur Spielraum für säkulare, nationale und sozialistische Ideen, die abseits der religiösen Fraktionierung der ägyptischen Gesellschaft Gemeinsamkeit schaffen sollten. ${ }^{6}$ Die religiöse Ausgrenzung der Kopten hat den Vorzug, dass ihre teilweise anders gearteten Normen auch für die Gesamtgesellschaft andere Gestalten von Sitte und Moral offen halten als die, die sich seit gut 30 Jahren mehr und mehr etabliert haben. So erzählte Andrea Nüsse von ihrer Begegnung mit einer 55 Jahre alten Muslima, die kein Kopftuch tragen mochte und trotz ihres Alters enge Jeans und Designerblusen liebte. Sie war es leid, in der Öffentlichkeit angegangen zu werden, weil sie kein Kopftuch trug. „Alte, du wirst bald sterben, es wäre besser, wenn du dein Haar bedeckst." Wo immer sie unterwegs war, ob in der U-Bahn oder beim Shopping, immer rechnete sie damit, dass sie ihres Äußeren wegen angepöbelt und zurechtgewiesen werden würde. So geht sie schon seit Jahren in die koptische Kirche zum Beten statt in die Moschee. „Hier kann ich in Ruhe sitzen, und der Gott ist ja ohnehin der Gleiche“, sagt sie. Sogar ein großes silbernes Kreuz hat sie sich gekauft und um den Hals gehängt. „Jetzt spricht mich niemand mehr an wegen meiner Kleidung, weil sie denken, ich sei Christin." Sie hat einen Weg gefunden, sich von dem sozialen Druck einer sich islamisierenden Gesellschaft zu be-

$\overline{4}$ Diese Textpassage und alle im Folgenden nicht gesondert ausgewiesenen stammen in teilweise überarbeiteter Form aus: M. Tamcke, Christentum im Land der Pharaonen, in: K. Pinggéra, Christentum im Schatten von Pyramiden und Minaretten. Beiträge zu Geschichte und Gegenwart der Koptischen Kirche, Hofgeismarer Protokolle 348, Hofgeismar 2009, 7-22.

5 Vgl. M. Tamcke, „Denn Glanz hat dieses verfälschte Gute übergenug“ - Wladimir Solowjews Weg mit den Kirchen und der Kirche, in: M. Delgado/G. Fuchs, Die Kirchenkritik derMystiker. Prophetie aus Gotteserfahrung, Band III: Von der Aufklärung bis zur Gegenwart, Studien zur christlichen Religions- und Kulturgeschichte 4, Fribourg/Stuttgart, 99-118.

6 Vgl. Tamcke, Christen, 122-151. 
freien. In der weithin islamisierten Welt Ägyptens ist der Lebensraum der Kopten noch eine Oase, in der nicht gelten muss, was ansonsten auf den gegängelten Menschen lastet und den Druck allem Nichtislamischen gegenüber erhöht.

\section{Historische und dogmengeschichtliche Blitzlichter}

Die Kopten in Ägypten nehmen für sich auch die Geschichte der griechischsprachigen Christenheit Ägyptens vor der konfessionellen Spaltung im 5. Jahrhundert in Anspruch. Als Emma Brunner-Traut ihre Monographie zu den Kopten schrieb, da erfasste sie die Geschichte der Kopten in altkirchlicher Zeit im Rückgriff auf vier besonders markante Personen der Geschichte der Alten Kirche und nannte die entsprechenden vier Unterkapitel zur Geschichte der Kopten: „Die ,Säule der Rechtgläubigkeit': Athanasios“, „Antonius und die Väter der Wüste“, „Pachom, der Gründer der Klöster“, „Schenute, Die Koptische Nationalkirche“.7 Die Kopten selbst führen ihren Ursprung auf Markus zurück. Den Kopten heilige Orte memorieren die Flucht der heiligen Familie nach Ägypten und deren Aufenthalt dort. So empfinden sich die Kopten nahe am biblischen Geschehen. Historisch gesicherte Nachrichten zu kirchlichen Strukturen besitzen wir erst für Bischof Demetrius von Alexandrien (um 180). Durch das Wirken von Klemens, Origenes und Athanasius wurde Alexandrien seit Ende des 2. Jahrhunderts zu einem Zentrum der altkirchlichen Theologie.

Die zahlreichen Märtyrer aus der Zeit der römischen Christenverfolgungen in Ägypten legten den Grundstein dafür, dass sich die Kirche bis heute als Märtyrerkirche versteht. Vieles, das dazu heute gesagt und gelebt wird und eigentlich die heutige Situation unter dem Druck der islamisierten ägyptischen Gesellschaft meint, tritt im Gewand historischer Erzählung zum 3. und 4. Jahrhundert auf (in der Kirche liegt der Zeitrechnung die Ära der Märtyrer zugrunde). Das war schon in den vorangehenden Jahrhunderten so.

Das Interesse an der zweitgrößten religiösen Gruppe des Landes ist nach wie vor eher gering. Die Koptische Orthodoxe Kirche oder gar auch noch die anderen Kirchen in ihrer lebendigen Vielfalt als blühende religiöse Gemeinschaften sind in den ägyptischen Schulbüchern kein Thema. ${ }^{8}$

$7 \quad$ Vgl. E. Brunner-Traut, Die Kopten: Leben und Lehre der frühen Christen in Ägypten, München 1993.

8 Vgl. K. Hock/J. Lähnemann, Die Darstellung des Christentums in Schulbüchern islamisch geprägter Länder, Bd 1: Ägypten und Palästina, bearb. von W. Reiss, Hamburg-Schenefeld 2005. 
In den christologischen Streitigkeiten des 5. Jahrhunderts vermochte sich der alexandrinische Patriarch Kyrill gegen Nestorius, den Patriarchen Konstantinopels, durchzusetzen.' Sein Nachfolger Dioskur unterlag dagegen auf dem Konzil von Chalcedon 451, nachdem er noch kurz zuvor die gesamte Kirche auf einem von den Vertretern Roms dann als Räubersynode betitelten Konzil auf eine theologische Grundlinie hatte nötigen können, der die Mehrheit der ägyptischen Gläubigen sich zugehörig fühlten. Das Konzil von 451 nahm mehrheitlich die Formel vom einen Christus in zwei Naturen an, während Dioskur an der alten Formel Kyrills von der eher die Einheit betonenden Natur des fleischgewordenen Wortes Gottes festhielt. Die unterschiedliche Haltung zu Chalcedon führte zu heftigen Auseinandersetzungen in der ägyptischen Christenheit, die auch eine ethnische Komponente hatten oder entwickelten: die griechische Minderheit stand schließlich der koptischen Mehrheit gegenüber. Ägypter zu sein, also Kopte zu sein, bedeutete fortan das Nein zu Chalcedon und das Ja zu Kyrill. Es bedeutete auch die zunehmende Verwendung des Koptischen anstelle des Griechischen. Dennoch nehmen die Kopten das Erbe der griechischen Kirchenväter Ägyptens ebenso für sich in Anspruch wie die Anhänger des griechischen Patriarchats von Alexandria. Gleiches gilt, der sozialen Abstammung vieler Mönche im frühchristlichen Ägypten tatsächlich entsprechend, für das Mönchtum. Ägyptische Christen sind stolz darauf, dass Ägypten die Wiege des christlichen Mönchtums war. Der Inhaber des Patriarchenamtes führt übrigens seit alter Zeit auch den Titel „Papst“.

Der Zusammenschluss der Gegner Chalcedons zu einer eigenen kirchlichen Organisation wurde gefördert durch das Wirken des antiochenischen Patriarchen Severus ( $† 538)$, der sich nach seiner Absetzung für 20 Jahre im Exil in Ägypten aufhielt. Die Abwendung von der Reichskirche verdrängte das Griechische als Liturgiesprache. Aus dem Mönchtum, wo schon von früh an das Koptische neben dem Griechischen in Gebrauch war, erwuchs schließlich der Meister der koptischen Schriftsprache, der vor 466 gestorbene Schenute von Atripe. In der theologischen Literatur des 6. und des 7. Jahrhunderts wächst der Anteil koptischer Werke beträchtlich. Die zunächst noch zwischen den Kirchenparteien strittigen Sitze der Hierarchen existierten bald doppelt: je einer für die Griechen und für die Kopten. Mit den dogmatisch ähnlich ausgerichteten Syrisch-Orthodoxen unterhielten die Kopten besonders enge Beziehungen.

$9 \quad$ Neben den eingangs aufgeführten historischen Darstellungen zur Koptischen Orthodoxen Kirche vgl. zu den dogmatischen Fragen und der Ökumene bes. D. W. Winkler, Koptische Kirche und Reichskirche, Innsbruck 1997. 
In den Jahrhunderten nach der islamischen Eroberung kam es zu schweren Bedrückungen des Volkes. Mehrere Koptenaufstände des 8. und des 9. Jahrhunderts wurden blutig niedergeschlagen. Allmählich wurden die Christen zur Minderheit. Die arabische Herrschaft über Ägypten (seit 642) hatte zur Folge, dass das Koptische als Umgangs- und Unterrichtssprache vom Arabischen abgelöst wurde. In dieser Sprache verfassten koptische Theologen zahlreiche Werke, die von einem durchweg hohen Bildungsniveau zeugen. Die koptischen Kirchenführer zogen sich in dieser Periode in die Wüstenklöster zurück. Im 11. Jahrhundert verlegte dann Patriarch Christodoulos seine Residenz dauerhaft nach Kairo und zollte damit den Veränderungen im Land seinen Tribut.

Im 12. Jahrhundert vereinheitlichen Reformen die Liturgie, Enzyklopädien fassten das entsprechende Wissen zusammen. Im 15. Jahrhundert war dieser Prozess der Vereinheitlichung abgeschlossen. Die Kopten waren über Jahrhunderte diejenigen, die wie keine andere christlich-orientalische Ethnie zur Blüte der christlich-arabischen Literatur beitrugen. Schon Georg Graf wies darauf hin, dass die Fülle dieses Schrifttums „namentlich in den nichtdogmatischen Disziplinen "beheimatet ist. Besonderer Reichtum findet sich in der religiösen Poesie.

Dogmatisch-theologische Werke verdanken sich oft erst der Herausforderung durch Kontrahenten. So begegnete der im 10. Jahrhundert lehrende Severus ibn al-Muqaffa, der Verfasser der grundlegenden "Geschichte der Patriarchen Alexandriens“ und des „Buches der Konzilien“, den Nöten, die das Verschwinden des Koptischen als Verkehrssprache zur Folge gehabt hatte. Die Gläubigen, der liturgischen Sprache nicht mehr mächtig, wurden sich nun vorrangig in der täglichen Unterhaltung mit Andersgläubigen ihres Glaubens inne. Dies führte zu starken Annäherungen an das muslimische Gottesbild. In seinem „Buch der Darlegung“ stellt Severus die zentralen Glaubensinhalte und das praktische Christentum dar. Einzigartigkeit und Trinität Gottes werden für Ungebildete und einfache Leute anhand von Gleichnissen dargelegt, Inkarnation und Kreuzigung werden vom Sündenfall ausgehend hergeleitet. Im moralischen Kampf gegen den Satan helfe die tägliche Teilnahme am liturgischen Gebet, und die sonntägliche Lesung der Heiligen Schrift, der miaphysitische Glaube wird gegen die Melkiten verteidigt, die Gläubigen zum Ertragen von Trübsalen ermutigt. Wir bewegen uns hier bereits in einem Feld, das trotz der Gründung des weltweiten internationalen Symposiums der christlich-arabischen Literatur erst ansatzweise von kritischer Forschung erschlossen ist. Bischof Paulus, ein Konvertit vom Islam zum Christentum, der den Quälereien durch seine Familie nach der Konversion durch Eintritt in ein Kloster ent- 
kommen war, steht da für viele Autoren, denen größere Aufmerksamkeit zukommen müsste, um das interreligiöse Bei-, Mit- und Nebeneinander in den spätmittelalterlichen Jahrhunderten differenzierter erfassen zu können. In der umfangreichen Literatur, die sich aus koptischer Sicht etwa mit dem Islam befasst, finden sich Argumente wie dasjenige, dass Mohammed nur für die Araber in der Zeit der Gahiliya gesandt worden und seine Aufnahme als Prophet für die Christen nicht notwendig sei. Dies sei durch den Quran selbst bezeugt. Oder die Unterredung eines koptischen Mönches mit drei Sufis über den Vorzug der Ehelosigkeit vor der Ehe. Kurzum, nicht immer wird der Weg herkömmlicher Apologetik beschritten, nicht immer werden die Araber als Strafe für eigene Sünde betrachtet. Patriarchen antworten den Einladungen Roms zur Union mit entsprechenden Darlegungen des Glaubens, so Johannes XI. (1428-1453) anlässlich der Einladung nach Florenz 1442. Doch als einflussreicher erwies sich da die antikatholische Polemik wie etwa die des Mönches von al-Baramus Ende des 19. Jahrhunderts.

\section{Reform und Erneuerung bei den Kopten}

Von dem Einmarsch der napoleonischen Truppen 1789 erhofften sich die Kopten ihre Freiheit und kämpften trotz der Warnungen von Patriarch Markos VIII. an der Seite der Franzosen. Nach dem Scheitern des Feldzuges kam es zu zahlreichen antichristlichen Gewalttaten. Unter Patriarch Kyrill IV. (1854-61) erfuhr das kirchliche Leben einen beachtlichen Aufschwung. Seine Reformen betrafen vor allen Dingen die Begründung eines modernen kirchlichen Unterrichtswesens. In der Amtszeit der folgenden Patriarchen kam es immer wieder zu Auseinandersetzungen zwischen reformfreudigen Laienorganisationen und der traditionell monastisch geprägten Hierarchie. Erst Patriarch Kyrill VI. (1959-1971) konnte die Kirche befrieden.

Obwohl in gewisser Weise um Ausgleich zwischen den Religionen bemüht, griffen die Engländer zur Festigung ihrer schwachen Position doch immer wieder auf die Kopten zurück. Fast schon symbolisch war es da, dass der 1908 zum Premierminister ernannte Kopte Boutros Ghali, ein Anglophiler, 1910 von einem Nationalisten ermordet wurde. Boutros Ghali $\left({ }^{*} 1846, \dagger 20.2 .1910\right)$ war Kind einer zur Oberschicht gehörenden koptischen Familie. Schon sein Vater stand im Dienst des ägyptischen Herrschers, des Khediven Ismail Pascha. Nach Sprachstudien und einer Anstellung bei der Handelskammer begann er über die Justiz eine politische Laufbahn. Über Ministerämter erlangte er schließlich 1908 das Amt des 
ägyptischen Ministerpräsidenten. Durch seine aktive Unterstützung der britischen Besatzungspolitik geriet er immer stärker in Gegensatz zu national gesinnten Kreisen. Insbesondere sein Verhalten bei der Verlängerung der Konzession des Sueskanals für die Briten um 40 Jahre trug dazu bei, ihn in breiten Kreisen der ägyptischen Bevölkerung unbeliebt zu machen. Im Februar 1910 wurde er bei einem Anschlag von einem jungen Medizinstudenten durch Schüsse aus einem Revolver getötet.

Die Briten ergriffen Maßnahmen, um der überproportionalen Präsenz der Kopten im Finanz- und Steuerwesen sowie im Verwaltungsapparat entgegenzuwirken. 1910 empfingen die Kopten immer noch $40 \%$ der Beamtengehälter Ägyptens. Zu dieser Zeit gehörte die Hälfte des gesamten ägyptischen Vermögens Ausländern, die fast durchweg Christen, viele auch orientalische Christen (Griechen und Armenier etwa) waren. Natürlich blieben die meisten Kopten Bauern und Handwerker, aber die hohe Präsenz im öffentlichen Dienst zeigte doch, wohin sich die koptische Bevölkerungsschicht bewegte. Beim großen Kongress der Kopten in Assiut 1911 forderten sie öffentlich einen ihnen gemäßen Anteil bei der Verwendung der Staatseinnahmen und eine weitere Öffnung der öffentlichen Ämter für sie sowie die Möglichkeit für Beamte und Schüler, am Sonntagsgottesdienst teilzunehmen und dafür von Dienst und Schule freigestellt zu werden. Der Erste Weltkrieg führte schließlich zur offiziellen Proklamation des britischen Protektorats über Ägypten. Im Zuge einer Studentenrevolte kam es dann zur Bildung einer ägyptischen Regierung, zu deren Leitung sich der ehemalige Finanzminister bereit erklärte. Es war wieder ein Kopte. In der ägyptischen Verfassung von 1923 wurde nochmals festgehalten, dass alle Ägypter vor dem Gesetz gleich seien. Patriarch Kyrill VI. stiftete Frieden für die innerlich gärende Kirche; es kam zur Gründung und Neubelebung von Klöstern, zur Intensivierung des geistlichen Lebens in den Pfarreien und zur pastoralen Betreuung der wachsenden Diaspora. Durch die relativ guten Beziehungen des Patriarchen zu Staatspräsident Nasser blieb das Verhältnis zum Staat erträglich. Der Präsident legte am 24. Juli 1965 den Grundstein für die St. Markus-Kathedrale in Kairo, und er nahm an der Einweihung der Kathedrale ebenso teil wie an den Feierlichkeiten zur 1900-Jahr-Feier des Heiligen Markus am 25. Juni 1968. Berühmt wurde das Diktum Nassers gegen die christlich-muslimischen Auseinandersetzungen in Ägypten: „Gott rief niemals zum Fanatismus, sondern zur Liebe auf.“ Allerdings darf dieses Verhältnis der jeweils Mächtigen zu den Kirchenführern der Kopten nicht als wirkliche Allianz verstanden werden. Eher ist dies die Art, wie Politik und Kirche in Ägypten miteinander bis zum arabischen Frühling diesen Jahres die alle betreffenden Probleme angingen und natio- 
nale Einheit auch da demonstrierten, wo man inhaltlich möglicherweise uneinig war.

Kyrill VI., der koptische Papst dieser Zeit, geboren am 12. August 1902, war nach der Zählung der Koptischen Orthodoxen Kirche der 116. Papst von Alexandrien und Patriarch des Stuhles vom Heiligen Markus. An ihm lässt sich gut zeigen, was die Koptische Orthodoxe Kirche bis heute prägt. Am 27. Juli 1927 war er ins Kloster Baramous eingetreten, 1928 wurde er dort zum Mönch geweiht. Die Mönchspriesterweihe erfolgte am 18. Juli 1931. Im Jahr 1932 zog er sich ins Einsiedlerleben zurück und lebte in der Höhle des Amba Sarabamon. 1936 verließ er seine Einsiedlerhöhle und ging nach Alt-Kairo. Ab 1942 versah er als Mönchspriester Pfarramtsdienste in Kairo. Als er 1944 ins Kloster Anba Samuel gerufen wurde, war das Ziel seiner Berufung dorthin die Neustrukturierung des Klosters. Auf seine Initiative hin und unter seiner Mitarbeit baute man zudem 1947 die Kirche St. Mina in Alt-Kairo. Am 10. Mai 1959 wurde er zum Patriarchen von Alexandria geweiht. In seine Zeit fiel die auch äußerlich greifbare weitere Verselbständigung der Kirche Äthiopiens, deren Patriarchen er 1959 weihte. Seine Verbundenheit mit den dortigen Christen belegen zwei Äthiopienreisen. Zur Eröffnung der Markuskathedrale 1968 fand die Reliquie des Hl. Evangelisten Markus von Italien zurück nach Ägypten ein symbolträchtiger Akt der Annäherung in ökumenischer und postkolonialer Hinsicht. Als Kirchenoberhaupt blieb er dem Mönchtum verbunden und ließ das Kloster St. Mina neu erbauen. Obwohl er selbst einer äußerst harten asketischen Disziplin anhing, wurde er zum Motor der Reform und suchte den steten Kontakt zu den Gläubigen durch zahlreiche Pastoralreisen.

Am 2. April 1968 hatte er eine Erscheinung der Hl. Maria Zeitoun. Über drei Jahre hinweg erschien Maria Zehntausenden von Zeitzeugen, Christen wie Muslimen. Wie in kaum einem anderen Ereignis bündelte sich hier, was bis heute die Frömmigkeit vieler Kopten auszeichnet. Damals waren zahlreiche Bekehrungen die Folge, Tausende unheilbar Kranke wurden geheilt. Das koptische Patriarchat erkannte die Erscheinung als echt an. ${ }^{10}$

Kyrill VI. starb am 9. März 1971 und wurde zunächst in Kairo in der Kathedrale, dann im Kloster St. Mina bestattet. Marienerscheinung, mönchisch-asketische Lebensweise, Reformen im Rahmen der Spiritualität, das liturgische Leben: Was diesen Patriarchen kennzeichnete, das charakteri-

10 Vgl. hierzu S. Keriakos, Les apparitions de la Vierge en Égypte: un lieu privilégié de la rencontre entre coptes et musulmans?, in: D. Albera/M. Couroucli, Religions traversées. Lieux saints partagés entre Chrétiens, Musulmans et Juifs en Méditerranée, Arles 2009, 255-294. 
siert das Leben der Gläubigen und der koptischen Kirche bis heute und gibt ihr ihr unverwechselbares Profil, das nicht in erster Linie ein dogmatisches, sondern eines von einer ausgeprägten Religiosität gekennzeichnetes ist.

Unter Schenouda III. (seit 1971) wurde dieses Erneuerungswerk konsequent fortgesetzt. Der ökumenische Dialog mit den chalcedonensischen Kirchen führte zur Verabschiedung christologischer Konsenserklärungen mit den Kirchen der byzantinischen Tradition und der römisch-katholischen Kirche. Die Gespräche zwischen den koptischen Protestanten und den koptischen Orthodoxen aber scheiterten, ebenso die zwischen den Kopten und den sogenannten „Nestorianern“. ${ }^{11}$

Eine Reform entfaltet hier ihre Wirkung, die Altes und Neues mischt und durch die Zeiten trägt. Sie bewahrt koptische Identität im Rückgriff auf eine starke Innerlichkeit, die ohne Umschweife auch die Traditionen des alten ägyptischen Mönchtums aus der Spätantike in die Neuzeit holt etwa in Gestalt der Sprüche der Wüstenväter, die als Einsiedler Ägyptens Wüsten bevölkerten und als charismatische Lehrer stark auf das Volk wirkten. Dies ist auch heute noch (oder besser: wieder) das Ideal mönchischer Spiritualität in Ägypten. So ist die Reform davon gekennzeichnet, einerseits ganz antik, andererseits ganz modern sein zu wollen.

\section{Die ambivalente Situation unter dem vergangenen Regime}

Die Situation heute ist völlig unüberschaubar. ${ }^{12} 2005$ standen bei den Parlamentswahlen nur zwei koptische Namen auf der Liste von 444 Kandidaten. Ein Theaterstück erregte in Alexandria die Gemüter, das von einem jungen Kopten berichtete, der zum Islam konvertiert und dann zum Christentum rekonvertiert. Den Islam stellte das Theaterstück eher in seinen negativen Seiten heraus. Islamische Demonstranten forderten eine Entschuldigung vom koptischen Patriarchen für die Aussagen des Stückes; mit dem Ein-

$\overline{11}$ Vgl. M. Tamcke/D. Heller, Was uns eint und was uns trennt. 5. theologische Konsultation zwischen der EKD und den Orientalisch-Orthodoxen Kirchen, Studien zur Orientalischen Kirchengeschichte 37, Münster 2005; M. Tamcke, Die Gespräche zwischen der EKD und den Orientalisch-Orthodoxen Kirchen, in: Erga 2005, Publications of Orthodox Theology in the University of Joensuu Nr. 35, Joensuu 2008, 123-144.

12 Weiterführende Literatur u. a.: M. Tamcke/M. Marten: Christian Witness Between Continuity and New Beginnings, Berlin 2006 (dort bes. die Beiträge von Burke und George); M. Tamcke, Daheim und in der Fremde, Hamburg 2002 (bes. die Beiträge von Heyer und Reiss zum Verhältnis von Koptischer und Eriträischer Orthodoxer Kirche, Meinardus zu Schenoudas Häresienlehre und Michaela Köger zum koptisch-orthodoxen Jugendbischofsamt); M. Tamcke, Koexistenz und Konfrontation, Hamburg 2003 (bes. Reiss zu Schulbüchern in Ägypten und dem koptisch-orthodoxen Jugendbischofsamt, Köger zum Bischofsamt für Öffentliche und Soziale Dienste, Meinardus zu übernatürlichen Erscheinungen). 
schreiten der Sicherheitskräfte eskalierte die Gewalt. Immer noch gibt es die mentale Haltung aus den Zeiten der Schutzverträge der früheren islamischen Herrschaft in Ägypten, nach denen das Oberhaupt der Christen haftbar zu machen ist für das, was unter den Christen vorgeht. Unterschieden wird da kaum zwischen Orthodoxen, Protestanten, Katholiken oder gar Sektierern. Ich erinnere mich an ein Gespräch mit dem gegenwärtigen Oberhaupt der Koptischen Orthodoxen Kirche, Papst Schenouda, dem es eine Last war, dass ihm in seinen Handlungen die Aktionen von freikirchlichen Missionaren, die besonders von Amerika aus unterstützt würden, immer wieder störend in den Weg gerieten. Übrigens greift das Theaterstück eine Problematik auf, die tatsächlich die ägyptische Wirklichkeit charakterisiert. So wurde der Fall einer 49-jährigen Frau publik, die Koptin und mit einem Christen verheiratet ist und mit diesem drei Kinder hat, die ebenfalls Christen sind. Die Frau wurde in der Hochzeitsnacht ihres Sohnes festgenommen. Sie sei, so lautete die Anschuldigung, eine Muslima, die mit einem Christen verheiratet sei. Der Hintergrund: ihr Vater war vor 30 Jahren zum Islam konvertiert, dann aber zum Christentum zurückgekehrt. Er hatte aber seine Papiere nicht ändern lassen. Daher wurde nun davon ausgegangen, dass der Vater Muslim geblieben sei und folgerichtig auch seine Tochter Muslima sei, was bedeutet, dass eine Ehe mit einem Christen nicht möglich ist. Aufsehen erregte auch der Fall eines zum Christentum konvertierten Scheichs, der am 6. April 2006 verhaftet wurde, weil er mit seiner Konversion den Islam beleidigt habe. Am 28. April 2007, nach zwei Jahren Haft, wurde er freigelassen. Die Gefängnisbeamten drückten ihm das Geld für das Taxi in die Hand, und der Mann fuhr heim, ohne zum Islam zurückgekehrt zu sein.

Ein Gesetz aus osmanischer Zeit, wonach das Staatsoberhaupt über den Bau oder die Renovierung einer Kirche zu entscheiden habe, schaffte der mittlerweile gestürzte Präsident Mubarrak erst im Jahr 2005 ab und verlagerte die Verantwortung auf die unteren Verwaltungsebenen. Beamtinnen ringen damit, dass ihnen das Kopftuch aufgenötigt wird, koptische Auslandsreisende berichten, dass sie in ihrem Pass einfach als Muslime eingetragen wurden und diesen Eintrag nur mit enormen juristischen Anstrengungen bei Gericht wieder revidieren lassen konnten.

Aber immer noch sind die Kopten besonders erfolgreich dort, wo sie nicht behindert werden. Als 1961 in Ägypten die Industrie verstaatlicht wurde, traf das besonders die Kopten: ihnen gehörten damals $51 \%$ der Banken, $44 \%$ der Industrie, $34 \%$ des landwirtschaftlich genutzten Landes. Noch immer machen die heute rund $10 \%$ Kopten $25 \%$ der Ärzte, Apotheker, Tierärzte, Journalisten und Rechtsanwälte aus, sind in Wirtschaft und 
selbstständigen Berufen überproportional erfolgreich. Das zeigt nach wie vor ihre große Aufgeschlossenheit gegenüber dem, was durch Bildung zu erlangen ist. Das hilft aber in einem Land extremer sozialer Spannungen zuweilen gerade nicht und dient manchen Propagandisten dazu, die Ursachen der Lage des Landes nicht beim Namen zu nennen, sondern die Christen als Sündenböcke für den Missstand von Land und Gesellschaft zu nutzen.

\section{Die Kopten und der "arabische Frühling“}

Seit Jahrhunderten waren und sind es die Christen im Orient gewöhnt, die Räume zu nutzen, die ihnen die muslimische Gesellschaft lässt, um an der Gestaltung der Gesellschaft im Lande teilzunehmen. Viele haben darin eine Meisterschaft entwickelt, wissen sozusagen schon intuitiv, was sie tun dürfen, ohne den Ärger der muslimischen Mitbewohner zu erwecken. Da ist eine gewisse Angepasstheit an die Welt der politisch etablierten Systeme im Orient nicht ausgeblieben. Die Menschen, die jetzt auf die Straße gehen und den Umschwung wollen, so sie Christen sind, wollen das nicht einfach mehr so hinnehmen. Am 16. April 2011 demonstrierten in Kairo 100.000 Kopten für Demokratie und Religionsfreiheit. Viele Muslime schlossen sich dem Marsch der Demonstranten an. Pater Mettias Nasr, der die Demonstration mitorganisierte, fasste das Ziel der Demonstration in wenigen Sätzen zusammen: „Wir wollen einen säkularen, demokratischen Staat und eine Verfassung ohne religiöse Klauseln. Und wir wollen Gesetze, die Diskriminierung verbieten. [...] Wir wollen allen Menschen zeigen, dass die Kopten präsent sind und faire und gesetzliche Forderungen haben." Anlass zur Demonstration war der 40. Gedenktag des Todes von neun Kopten am 9. März während der Erstürmung eines Viertels in einem Kairoer Vorort. In der noch unklaren Situation, in der viele Kräfte miteinander darum ringen, wohin das Land in $\mathrm{Zu}$ kunft steuern soll, wollen die Kopten sich nicht einfach unsichtbar machen und die Entscheidungen den anderen politischen Kräften im Land überlassen. Sie wollen mitgestalten. Sie hoffen auf eine bessere Zukunft, auf bessere Lebensbedingungen, auf echte religiöse Gleichberechtigung. Sie halten an dieser Vorstellung fest, obwohl ihnen Gegendruck widerfährt, obwohl sich Ausschreitungen auch gezielt gegen sie gerichtet haben. So wollen sie den Geist des Frühlings in der arabischen Welt weiter tragen - über die Abgründe schmerzlicher Gegenerfahrungen hinweg. Auf dem TahrirPlatz hatten sie mit Muslimen Seite an Seite gestanden, dort kam es zu Verbrüderungsszenen zwischen Christen und Muslimen mit zum Himmel 
gestreckten Kreuzen und Koranen. Ein neues Miteinander schien möglich zu werden. Aber schon am 10. März kam es zu so massiven Auseinandersetzungen zwischen Christen und Muslimen in Kairo, dass 10 Menschen starben und 110 verletzt wurden. Sechs der 10 Toten waren Christen. Initialzündung der Konfrontation war ein Brandanschlag auf eine Kirche im Süden Kairos gewesen. 1.000 Kopten, die dagegen Stellung bezogen, gerieten ins Visier von Bewaffneten. Die toten Kopten waren an Schussverletzungen gestorben. Gewalt scheint für die Kopten die Reform begleiten zu sollen, wie sie auch am Anfang des Prozesses gestanden hatte, als in der Neujahrsnacht in der Markus-und-Petrus-Kirche in Alexandria eine durch einen Selbstmordattentäter verursachte Explosion 21 Gläubige mit in den Tod riss und viele verwundete. Koptische Jugendliche zimmerten aus Latten überlebensgroße Kreuze und riefen: „Unser Leben, unsere Seele geben wir für unser Kreuz!“ Steine flogen gegen die gegenüberliegende Schark-al-Medina-Moschee, Sonderpolizei traf ein und drängte die Protestierenden mit Tränengas ab. Es wäre wenig realistisch, von den koptischen Jugendlichen zu erwarten, dass sie in einem solchen Moment nicht ihrer ohnmächtigen Wut Luft machten.

Hier beginnt das innere Problem. Viele, die in den Kirchen Verantwortung tragen, sorgen sich um die Ruhe im Land und betonen, dass sie sich politisch abstinent verhalten, aus Sorge, dass eine politische Parteinahme sie im öffentlichen Leben angreifbar machen würde. Schon lange wird die Kritik an politischen Missständen eher im eigenen Milieu geäußert als wahrhaftig im Dialog mit den konkurrierenden Kräften im Land. So kennt jeder, der in der Region etwas beheimatet ist, jenes schizophrene Verhalten, dass äußerlich gute Miene zu bösem Spiel gemacht, aber im vertrauten Zirkel unverblümt mit der anderen Seite in deren Abwesenheit abgerechnet wird.

Die zur Reform der Verfassung eingesetzte Kommission im Land mag das verdeutlichen. 11 Mitglieder hat sie, davon ist nur ein Mitglied Christ, der 57jährige Professor für internationales Recht und Vorsitzende der Union ägyptischer Menschenrechtsgruppen, Naguib Gobraiel. Ein anderes Mitglied gilt als Vertreter der Muslimbruderschaft. Die Hauptforderung der Kopten ist die Herstellung der Religionsfreiheit und die Streichung des Paragraphen 2 der Verfassung, der die Scharia als wichtigste Quelle des Rechts in Ägypten festhält. Sie wollen einen religiös neutralen Staat, um endlich die Benachteiligung infolge der Konversionsthematik etwa zu überwinden. Im Verfassungsrat sitzt selbstverständlich ein Vertreter der international führenden Religionshochschule, der Al Azhar. Aber es sitzt dort kein Bischof einer Kirche. Naguib Gobraiel dazu: „Wir sind sehr enttäuscht 
von der Leitung unserer Kirche. Zu der Protestwelle im Land hat sie keine Stellung genommen. Papst Schenouda III. hat bisher kein Wort der Ermutigung gesagt an die Adresse der Jugend. Er will unter allen Umständen den Eindruck vermeiden, die koptische Kirche mische sich politisch ein." Übrigens gesteht Gobraiel offen seine Angst ein vor den Muslimbrüdern und hält deren derzeitig moderates Verhalten für ein taktisches Spiel.

Die Frage, wie weit sich die offizielle Kirche politisch im Geschehen positionieren darf, lastet schwer auf dem Verhältnis der Vorwärtsdrängenden zu denen, die gezeichnet sind von den historischen Erfahrungen und die sich von daher die Maximen ihres Nichthandelns und Stillhaltens bestimmen lassen. Die Entwicklung derzeit ist gekennzeichnet von Widersprüchlichkeiten. Erst die kommenden Jahre werden zeigen, was der politische Umbruch für die Christen in Ägypten und die Religionsfreiheit bedeuten wird.

\section{Ausblick: Die anderen Kirchen in Ägypten}

1) Ägypten war Teil des Römischen Reiches. Hier war Alexandria, ein Zentrum griechischer Gelehrsamkeit, hier wirkten berühmte griechischsprachige Kirchenväter wie Athanasius oder Clemens im Rahmen der Kirche des Römischen Reiches. Als es im 5. Jahrhundert über der christologischen Frage zum Zerbrechen der kirchlichen Einheit kam, was auch ein Bruch zwischen Griechen und Kopten in Ägypten war, verblieben die Griechen bei der Reichskirche. Alexandria gehört auf der Liste der Patriarchate der dritte Rang nach Rom und Konstantinopel. Das griechische Patriarchat war stärker von der arabischen Eroberung betroffen als das der konkurrierenden Kopten. In osmanischer Zeit hielten sich viele Patriarchen überwiegend oder auch ganz in Konstantinopel auf, so auch der spätere Ökumenische Patriarch Kyrill Loukaris, der wegen seiner calvinistischen Tendenzen zu leiden hatte und sich in Ägypten als Reformer hervortat. Erst 1846 kam mit Hierotheos I. der orthodoxe Patriarch von Alexandria wieder dauerhaft nach Ägypten zurück. Im letzten Viertel des 19. Jahrhunderts geschah eine massive Einwanderung von Griechen nach Ägypten. ${ }^{13}$ Die meisten der 250.000 Griechen, die daraufhin in Ägypten lebten, verließen das Land in den Jahrzehnten nach der Revolution 1952 wieder. Sie hatten zu den Hauptverlierern der politischen Umwälzungen gehört. Heute sind es wohl nur 5000 bis 10.000 Griechen, die zum Patriarchat gehören, daneben ca. 10.000 christliche Araber. Wer dem Lebensgefühl dieser grie-

13 A. Kitroeff, The Greeks in Egypt 1919/37, London 1989. 
chischen Minderheit nachspüren will, der kann auch hier in Deutschland auf die Übersetzung des Werkes von Konstantinos Kavafis zurückgreifen, jenem homosexuellen Dichter, den Forster in Alexandria besuchte und der nun weltweit zu einer Legende für das Morbide des Griechischen in Ägypten wurde - zum Repräsentanten einer Kultur, die nur noch ein Schatten ihrer selbst ist. Utensilien wie seine Brille bekommt man im Museum gezeigt, das nun in Alexandria als gewaltiger Bau bewusst irgendwie an die Geschichte der berühmten Bibliothek von Alexandria anknüpft. Das Patriarchat ist aber für die gesamten orthodoxen Missionskirchen auf dem afrikanischen Kontinent zuständig, mit 18 Metropolien und 6 Bistümern über ganz Afrika verstreut, die ca. 300.000 Gläubigen dienen.

Nachdem sich die orthodoxen Kirchen der byzantinischen Tradition mit den orthodoxen Kirchen der orientalischen Tradition Anfang der 90er Jahre des vergangenen Jahrhunderts über die sie trennende theologischen Kernfrage im Bereich der Christologie geeinigt hatten, kam es auch in Ägypten zu Schritten ökumenischer Verständigung. So einigten sich die beiden Patriarchate am 5. April 2001 auf ein Papier zu Trauung und Ehefragen. Es sei künftig nicht mehr erforderlich, dass die Paare in Kirchen beider Patriarchate getraut würden. Es genüge die Zeremonie in einer der Kirchen, die dann auch für die andere gültig vollzogen sei. Trotzdem hält der Protest gegen die Annäherung der beiden orthodoxen Kirchenfamilien bis heute auch in Ägypten an. Ich fand etwa einen Kommentar zu dem im Internet veröffentlichten Dokument, der schlicht in dem Satz gipfelte: „This document officially requires the clergy of the Patriarchate of Alexandria to accept a lie." Dennoch: die moderne Ökumene hat auch in Ägypten in den letzten Jahrzehnten manches bewegt, was den heillosen Streit zumindest notdürftig überbrückt.

2) Für die Armenier war Ägypten lange Zeit eine Region, in der sie sich vor den Verfolgungen in Sicherheit bringen konnten, mit denen sie im Osmanischen Reich überzogen wurden. Hier sammelten sich unzählige armenische Flüchtlinge, die den Nachstellungen im Osmanischen Reich entkommen waren. In den armenischen Lagern wirkten noch viele Jahre nach dem Ersten Weltkrieg und dem Tiefpunkt armenischer Präsenz besonders im osttürkischen Raum zahlreiche, auch deutsche und schweizerische Hilfswerke an der Rettung der Überlebenden des Völkermords mit. Die Diözese der Armenier in Alexandria soll heute aber nur noch ca. 2.000 Armenier umfassen. Die meisten, die in der Zeit des Ersten Weltkrieges nach Ägypten geflohen waren, sind in den Jahrzehnten danach weitergezogen nach Amerika oder in andere Teile der Welt. 
3) Die maronitische Diözese von Kairo soll nur noch 5.000 Gläubige zählen. Auch den Maroniten des syrisch-libanesischen Raumes war Ägypten ein Land der freieren Lebensart, solange sie seit der Mitte des 19. Jahrhunderts unter Verfolgungen litten. Ihren Namen führen sie auf einen syrischen Mönch aus altkirchlicher Zeit zurück. Eine christliche Libanesin war die erste Frau, die in Ägypten öffentlich als Studentin den Vorlesungen folgte. Solche christliche Gruppen alter orientalischer Kirchen erinnern daran, dass Ägypten in der jüngeren Geschichte bis zur Mitte des 20. Jahrhunderts ein Raum war, in dem Christen ungestörter als anderswo im Orient ihren Glauben ausleben durften. Stark eingeschränkt gilt das noch heute etwa für die schwer verfolgten Christen Sudans, die sich zeitweilig in Lagern in Ägypten sammelten und hier erste Hilfe erfuhren.

4) Die größte der hier vorgestellten „anderen Kirchen“ ist die Koptische Evangelische Kirche von Ägypten. Ihre Wurzeln liegen in der Mission der amerikanischen Presbyterianer. 1863 gründeten diese ein Seminar in Kairo. Seit 1957 ist die Kirche unabhängig und nicht mehr Teil der Koptischen Orthodoxen Kirche. Die Angaben zu den Mitgliederzahlen schwanken erheblich und werden mit 200.000 bis 500.000 angegeben. Die Nilsynode hat heute ca. 300 Gemeinden. Die Kirche sticht durch ihre überproportionale diakonische Aktivität und ihr Engagement im Bildungsbereich hervor. Glaubt man Heather J. Sharkeys gerade erschienener Monographie zur Frühzeit dieser Kirche in Gestalt der amerikanischen Mission, dann hätten die Missionare neue Modelle der Bürgerschaft und v. a. der Rolle der Frau mit sich gebracht. ${ }^{14}$ Stimulierend wirkte sich die missionarische Tätigkeit besonders im Bereich sozialer Aktivitäten in Ägypten aus, wo die Notwendigkeit derartiger Anstrengungen nun auch von anderen gesellschaftlichen Kräften aufgenommen und andererseits den missionarischen Aktivitäten Beschränkungen auferlegt wurden. Da die Missionserfolge unter Muslimen klein blieben, rekrutierten sich die Gläubigen zumeist aus den Reihen der orthodoxen Kirchen im Land.

5) Eher klein sind die Gemeinden der Anglikaner in Ägypten, deren Diözese von Ägypten auch Nordafrika (Gemeinden existieren beispielsweise in Libyen, Tunesien und Algerien), Äthiopien, Eriträa, Somalia und Djibouti umfasst. Seit 1945 ist der Sudan eine eigenständige Diözese dieser Kirche. Mit 35.000 getauften Mitgliedern wird ihre Zahl angegeben, wel-

14 H. J. Sharkey, American Evangelicals in Egypt. Missionary Encounters in an Age of Empire, Princeton 2008. 
che in 55 Gemeinden organisiert sind, in denen 90 Pfarrer ihren Dienst versehen. Erst 1974 wurde der erste Ägypter in dieser Kirche zum Bischof geweiht. Bis dahin hatten Briten dieses Amt versehen. Spektakulär war die Zerstörung der anglikanischen Kathedrale Aller Heiligen in Kairo 1970 zugunsten des Baus einer Nilbrücke. Elf Jahre, von 1977 bis 1988, dauerte der Bau der neuen Kirche, die besonders den Flüchtlingen aus dem Sudan dient: $80 \%$ ihrer Mitglieder sind sudanesische Flüchtlinge.

6) Methodisten, Baptisten, Assemblies of God, Plymouth Brethren - all diese Kirchen entwickelten sich im 20. Jahrhundert ebenfalls in Ägypten. 1921 einigten sich die wichtigsten protestantischen Kirchen darauf, zusammenzuarbeiten. 1952 beendete die Revolution alle missionarische Arbeit, doch blieben 17 protestantische Kirchen als selbstständig in Ägypten zurück. Sie sind zusammengefasst im Protestant Council, das sie gegenüber der Regierung vertritt. 12 der 20 Mitglieder des Protestant Council gehören der Koptischen Evangelischen Kirche an, da sie die größte der 17 protestantischen Kirchen Ägyptens ist. Alle diese Kirchen entstanden auf dem Boden der Koptischen Orthodoxen Kirche. Da sich die Konversion von Muslimen schon aufgrund der gesellschaftlichen Restriktionen als äußerst schwierig erwies, ganz abgesehen von der Reaktion der muslimischen Religionsgemeinschaft aufgrund ihrer Haltung zur Abwendung vom Islam (Apostasie), ist das Verhältnis vieler dieser Kirchen zur Mutterkirche oft nicht einfach.

7) Deutlich jünger als die presbyterianische Kirche in Ägypten ist die Koptisch-Katholische Kirche. Zwar hatte es auf dem Konzil von Florenz am 4. Februar 1442 den Versuch gegeben, die Kopten der Römisch-Katholischen Kirche anzugliedern, doch blieb der dort ausgehandelte Unionsvertrag ohne ernsthafte Resonanz. 1630 begannen die Kapuziner ihre Mission in Kairo, 1675 die Jesuiten. 1741 trat der koptische Bischof von Jerusalem zeitweilig zur Römisch-Katholischen Kirche über, 1824 erfolgte ein vergeblicher Versuch, katholischerseits wieder in Ägypten präsent zu sein. 1895 errichtete dann Papst Leo XIII. erneut das unierte Patriarchat, dessen erster Patriarch bereits 1908 wieder seinen Dienst aufgeben musste. 1893 übergaben die Franziskaner in Ägypten der Koptisch-Katholischen Kirche zehn Gotteshäuser, nachdem die Osmanen bereits 1829 den koptischen Katholiken den Bau eigener Kirchen gestattet hatten. Seit 1908 herrschten Kontroversen über die damals eingeführten lateinischen Riten in der Kirche, und bis 1927 blieb das Amt des Patriarchen vakant. 1947 erfolgte die erneute Errichtung. Dies war die Geburtsstunde der Koptisch- 
Katholischen Kirche in ihrer heutigen Form. 160.000 bis knapp 200.000 Gläubige zählen zu dieser Kirche. Der vor zwei Jahren gewählte Patriarch führt den Titel „Patriarch von Alexandria“.

8) So manches Missionswerk ist, ohne bleibende Spuren zu hinterlassen, nach Jahren oder Jahrzehnten der Wirksamkeit in Ägypten schlicht wieder verschwunden. Das schmälert nicht unbedingt seine Leistungen. Es hatte schon vor den Amerikanern Missionsbemühungen von Protestanten in Ägypten gegeben. In der Mitte des 18. Jahrhunderts wirkten dort die Herrnhuter. Dazu erarbeiten wir gegenwärtig eine Edition der wichtigsten Quellen, die einen ungewohnten Einblick geben in die Welt Ägyptens und die Begegnung von Deutschen und Kopten in vornapoleonischer Zeit. Die ersten Bände stehen zur Publikation an, nur fehlt noch das Geld für die Drucklegung.

Bei den Herrnhutern entstand auch die erste arabische Übersetzung der Confessio Augustana (viele andere Schriften folgten, darunter besonders Übersetzungen von Briefen des Grafen Zinzendorff an den koptischen Papst und dessen Briefe an den Grafen). Von 1815 bis 1849 versuchte sich dann die englische Church Missionary Society hier. Deutsche machten sich gar daran, Missionsstationen auf einer so genannten Apostelstraße quer durch Ägypten bis nach Äthiopien zu gründen, Kaiserswerther und Johanniter gründeten Krankenhäuser und Schulen, und hier lag auch der Schwerpunkt der Sudan-Pioniermission, die eigentlich auf den Sudan zielte und heute noch unter anderem Namen in der Region arbeitet. Besonders die herausstechenden Aktivitäten der Herrnhuter führten doch unmittelbar zu keiner Kirchengründung. Das Anliegen der Herrnhuter war ohnehin die Belebung der Koptischen Orthodoxen Kirche gewesen, bei bewusstem Verzicht auf die Sammlung eigener Gemeinden, die aus der Koptischen Orthodoxen Kirche hätten herausgeführt werden müssen. Schöne Zeugnisse zwischenkirchlicher Verständigung sind die arabischen Briefe des Patriarchen Markos an und für die Herrnhuter. So empfahl er dem äthiopischen Abuna den Missionar Hocker ohne Einschränkung: „Überbringer dieses ist der gesegnete Sohn und ehrwürdige Diaconus Irenaeus Ockar, ein gesegneter Mensch, von Geschlecht ein Francke, der alle Christen und alle Creatur Gottes lieb hat." 